@( $($ How to eliminate tuberculosis 3

\title{
Controlling the seedbeds of tuberculosis: diagnosis and treatment of tuberculosis infection
}

\author{
Molebogeng X Rangaka, Solange C Cavalcante, Ben J Marais, SokThim, Neil A Martinson, Soumya Swaminathan, Richard E Chaisson
}

Lancet 2015; 386: 2344-53

Published Online

October 26, 2015

http://dx.doi.org/10.1016/

S0140-6736(15)00323-2

This online publication has

been corrected. The corrected

version first appeared at

thelancet.com on Dec 3, 2015

See Comment pages 2231,

e46, and e48

This is the third in a Series of

four papers about how to

eliminate tuberculosis

Institute of Epidemiology and

Health, University College

London, London, UK and

Department of Medicine, Institute of Infectious Disease

and Molecular Medicine,

University of Cape Town, Cape

Town, South Africa

(MX Rangaka PhD); Evandro Chagas National Institute of

Infectious Diseases, Rio de Janeiro, Brazil

(S C Cavalcante PhD); Children's Hospital at Westmead and the Centre for Research Excellence in Tuberculosis, University of Sydney, Australia (B) Marais PhD); Cambodian Health Committee, Phnom Penh, Cambodia (S Thim MD): Perinatal HIV Research Unit, University of Witwatersrand,

Soweto, South Africa

(N A Martinson MBBCh); National Institute for Research on Tuberculosis, Chennai, India

(S Swaminathan $\mathrm{PhD}$ ); and

Center for Tuberculosis

Research, Johns Hopkins University School of Medicine, Baltimore, MD, USA (RE Chaisson MD)

Correspondence to: Richard E Chaisson, Center for

Tuberculosis Research, Johns

Hopkins University School of Medicine, Baltimore, MD 21287,

rchaiss@jhmi.edu

The billions of people with latent tuberculosis infection serve as the seedbeds for future cases of active tuberculosis. Virtually all episodes of tuberculosis disease are preceded by a period of asymptomatic Mycobacterium tuberculosis infection; therefore, identifying infected individuals most likely to progress to disease and treating such subclinical infections to prevent future disease provides a crucial opportunity to interrupt tuberculosis transmission and reduce the global burden of tuberculosis disease. Programmes focusing on single strategies rather than comprehensive programmes that deliver an integrated arsenal for tuberculosis control might continue to struggle. Tuberculosis preventive therapy is a poorly used method that is essential for controlling the reservoirs of disease that drive the epidemic. Comprehensive control strategies that combine preventive therapy for the most high-risk populations and communities with improved case-finding and treatment, control of transmission, and health systems strengthening could ultimately lead to worldwide tuberculosis elimination. In this Series paper we outline challenges to implementation of preventive therapy and provide pragmatic suggestions for overcoming them. We further advocate for tuberculosis preventive therapy as the core of a renewed worldwide focus to implement a comprehensive epidemic control strategy that would reduce new tuberculosis cases to elimination targets. This strategy would be underpinned by accelerated research to further understand the biology of subclinical tuberculosis infections, develop novel diagnostics and drug regimens specifically for subclinical tuberculosis infection, strengthen health systems and community engagement, and enhance sustainable large scale implementation of preventive therapy programmes.

\section{Current situation and rationale for change}

The control of an infectious disease epidemic requires active case detection, treatment when possible, interruption of transmission, and enhancement of immunity for susceptible individuals. If elimination is desired, containment of the reservoirs, or seedbeds, of infection is essential. Tuberculosis is a disease whose pathogenesis is characterised by a period of asymptomatic subclinical infection that might last for weeks to decades; as a result, a large reservoir of infected human beings exists, among whom new cases might arise at any time. Although

Key messages

- Latent tuberculosis infection serves as the seedbed for virtually all new cases of active tuberculosis disease and should be addressed as an essential part of tuberculosis elimination.

- The efficacy and effectiveness of treating latent tuberculosis infection have been known for more than 50 years, but policies have not emphasised the epidemiological effect of treating these infections.

- Several clinical, administrative, and policy constraints have restricted use of preventive therapy.

- Populations at highest risk of progression from latent to active tuberculosis can be identified, and diagnostic tests and risk stratification can be used to select those individuals most likely to benefit from preventive therapy.

- New regimens for treating latent tuberculosis can simplify, shorten, and improve adherence to preventive therapy. aggressive strategies to find and treat all cases of disease are necessary to turn the tide of the worldwide tuberculosis epidemic, these strategies alone will not be sufficient to end tuberculosis by WHO's 2035 target because they do not address the large existing reservoir of infection. The WHO policy now recognises that stopping the tuberculosis pandemic will require unprecedented efforts to address the human seedbeds of disease. ${ }^{1}$

Contemporary understanding of what has long been called latent tuberculosis infection has evolved. Rather than a binary distinction between latent and active states, tuberculosis infection is now understood as a dynamic multistate gradient of latent subclinical infection to clinically active disease; a process that is imperfectly represented by the dichotomous classification. ${ }^{2-4}$ The spectrum of tuberculosis infection ranges from individuals who mount effective immune responses that eradicate all viable bacilli, to those whose responses contain the infection but who continue to harbour populations of bacilli that intermittently replicate in macrophages, granulomata, and other tissues, engaged in an intricate struggle with the host immune system. ${ }^{5}$ There are also those with no effective immunity against tuberculosis who progress rapidly from tuberculosis infection to disease, such as young children, the chronically ill, and, HIV-infected individuals., ${ }^{2,4}$ Differences in host immune responses affect the risk of tuberculosis infection progressing to active disease.

The population dynamics of tuberculosis begin with subclinical asymptomatic infections from which active cases arise (figure); these infections then spread to 
contacts (individuals in contact with the infectious case). Newly infected contacts might then progress to active tuberculosis disease, a process that could take weeks to more than a year, or enter the large pool of asymptomatically infected individuals and be at risk of future tuberculosis. Tuberculosis case-finding and treatment of disease prevents the spread of tuberculosis by reducing the number of secondary infections resulting from each new case, but this strategy alone cannot lead to elimination of the disease. ${ }^{6}$ The vaccine available at present mostly mitigates disease severity in infants and young children, and despite high levels of coverage in many countries, it has not had an appreciable populationlevel effect on the incidence of pulmonary tuberculosis in adults worldwide. The only strategy for preventing new cases from arising in individuals sufficiently exposed to tuberculosis is to give treatment to those exposed to or infected by $M$ tuberculosis, which prevents progression to active disease in the newly or remotely infected. ${ }^{4,7}$ Thus, treatment of latent tuberculosis infection (called preventive therapy to differentiate it from treatment of active disease, which requires multidrug therapy) is an essential component of the strategy for elimination of tuberculosis, yet it is the least exercised option of all of the proven methods for combatting the worldwide epidemic because it has been underemphasised in disease control policies for several decades.

In the 1990s, Dye and colleagues estimated that a third of the world population was infected with $M$ tuberculosis, ${ }^{8}$ and a study from China found that a quarter to a third of adults in rural areas had tuberculosis infection, ${ }^{9}$ emphasising the enormous reservoir that serves as the seedbed for new cases of active tuberculosis. Identification of high-risk epidemic locales (see Theron et al, paper 1 ) $^{10}$ as well as intensification of case-finding and improvements in treatment (see Yuen et al, paper 2 ), ${ }^{11}$ although important, cannot alone achieve tuberculosis elimination because the reservoir of asymptomatically infected individuals will continue to produce millions of new cases of reactivation tuberculosis for decades to come. Even a highly effective new vaccine that prevents disease after new infection would not be sufficient for eliminating tuberculosis because the high prevalence of existing infections would not be affected. Thus, epidemiologically sound tuberculosis elimination strategies should include treatment of tuberculosis infection to be effective. ${ }^{12}$

Provision of treatment to prevent the establishment of a productive infection or progression of infection to disease is an established strategy for the control and elimination of major infectious diseases of public health relevance. For instance, eradication of smallpox was possible through a worldwide multipronged strategy to limit transmission that included finding and offering vaccination to contacts of infected people residing in epidemic hotspots. ${ }^{13}$ Additionally, mass preventive therapy has been used to combat both Chlamydia trachomatis and Onchocera volvulus. ${ }^{14}$ Because the human

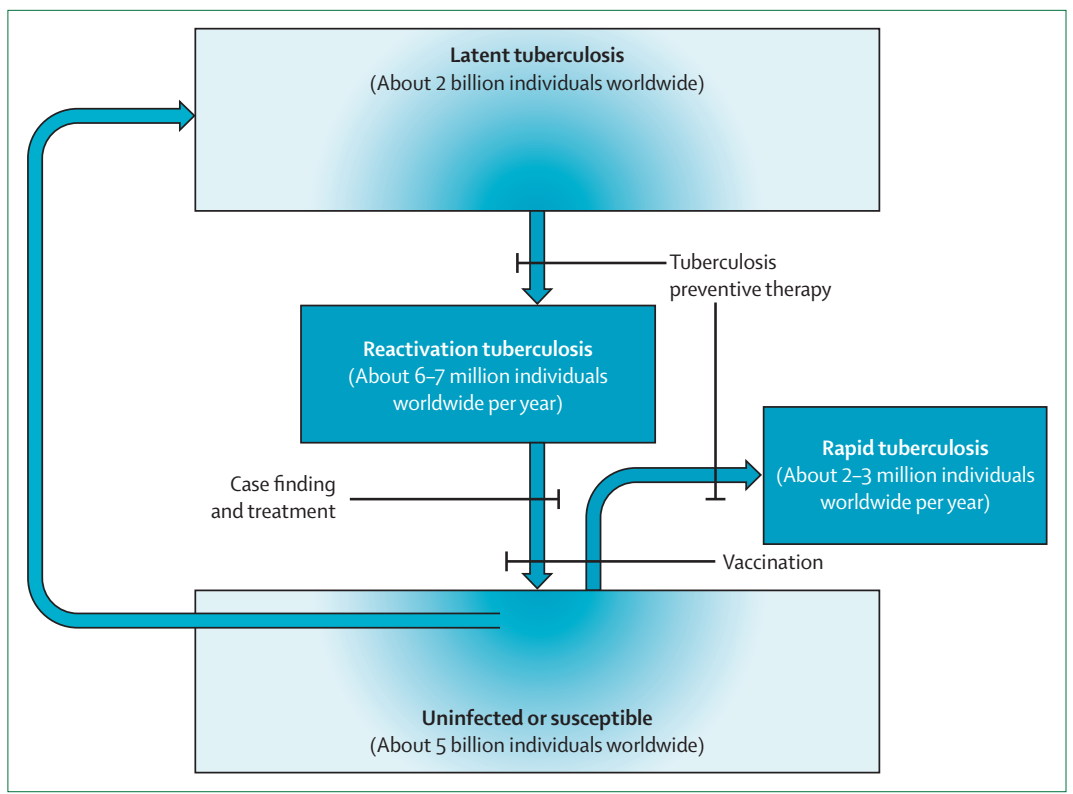

Figure: Population-level control strategies for tuberculosis elimination.

Arrows show the dynamics of $M$ tuberculosis in the world's population, with flow from latent infection to active disease, transmission to new hosts, followed by either rapid progression to disease and ongoing transmission or entry into the pool of latent infections. Bars show how different control measures affect these dynamics, interrupting the chain of events. Even if diagnosis and treatment of active tuberculosis is maximised and a new effective vaccine is developed, reactivation from the billions of latently infected will result in new cases for decades to come.

reservoir of $M$ tuberculosis infection is enormous, overwhelmingly asymptomatic, and long-lived, a strategy to identify individuals who are at highest risk of progression to disease, who would thus benefit the most from preventive therapy, is widely recommended.

Some groups of people are at increased risk of progression to disease. These include close contacts of people with active tuberculosis disease, young children, elderly people, and people with HIV or other immunodeficiencies. Additionally, although tuberculosis infection tests available at present are imperfect proxies of risk, the Mantoux tuberculin skin test has been prospectively assessed to predict benefit from preventive therapy in several settings and populations.? Less robust evidence exists for the newer interferon $\gamma$ release assays, though it is likely to be as predictive. ${ }^{15}$

The benefits of tuberculosis preventive therapy have been known for more than 60 years. Pioneering studies in the 1950s-60s provided overwhelming evidence of the efficacy of isoniazid preventive therapy in preventing active tuberculosis in children, Alaskan Native populations, residents of congregate living facilities such as mental hospitals, and household contacts of tuberculosis patients. ${ }^{7,16-18}$ Subsequent work has further documented benefits of preventive therapy for individuals with evidence of recent infection, those with radiographic evidence of previous untreated tuberculosis, people with HIV infection, recipients of immunosuppressive therapy such as TNF- $\alpha$ inhibitors, and other immunocompromised individuals. We summarise the populations at 
risk for tuberculosis who should benefit from preventive therapy, tests for tuberculosis infection, and available options for treatment (panel 1).

Despite abundance of evidence of its efficacy, use of preventive therapy outside North America has been restricted for the past 40 years because tuberculosis control programmes have focused almost exclusively on detection and treatment of infectious tuberculosis cases. Preventive therapy has been patchily targeted at children aged 5 years or younger with household exposure to an infected person. Although most countries have formal policies recommending treatment of these individuals, implementation in countries with a high tuberculosis burden has been near absent. Additionally, few of these countries have historically had policies addressing other high-risk individuals. WHO first recommended isoniazid preventive therapy for people living with HIV as a personal health measure in 1998 , and updated this to a public health recommendation in $2010 .{ }^{19}$ However, of the

Panel 1: Populations and individuals who benefit from tuberculosis preventive therapy, testing strategies, and treatment regimens

\section{Populations at increased risk}

- Residents of or immigrants from high-burden areas

- People with HIV infection

- Contacts of infectious cases

- Recent tuberculin skin test (TST) or interferon $\gamma$ release assay (IGRA) converters

- Recipients of TNFa blockers

- Recipients of immunosuppressive therapy or transplantation

- Residents of congregate living facilities including prisons

- Homeless people

- People with diabetes

- Cigarette smokers

- Miners and people with silicosis

- Residents of congregate living facilities

- Health-care workers and people who visit health-care facilities (in high tuberculosis burden areas)

\section{Tests for tuberculosis infection}

- TST

- $\quad \geq 5 \mathrm{~mm}$ induration at 2-3 days deemed positive for HIV-infected individuals, close contacts of cases, and young children

- $\quad \geq 10 \mathrm{~mm}$ induration at 2-3 days deemed positive for other risk categories

- $\quad \geq 15 \mathrm{~mm}$ induration at 2-3 days for those with no identifiable risk factors

- IGRA

- Quantiferon-Gold In Tube Assay (QGIT): $>0.35 \mathrm{IU} / \mathrm{mL}$ deemed positive

- T-SPOT Test: $>8$ spot-forming cells deemed positive

- Proxy measures of tuberculosis infection when testing unavailable

- Household contact with a pulmonary tuberculosis case

- Resident of areas with high burden of latent tuberculosis

Treatment regimens for tuberculosis infection

- Isoniazid daily or twice weekly for 6, 9, 12, or 36 months

- Rifampicin daily for 3-4 months

- Rifampicin and isoniazid daily or two to three times per week for 3 months

- Rifapentine and isoniazid once weekly for 12 weeks
22 high tuberculosis-burden countries, only South Africa and Brazil have ambitious national policies to provide preventive therapy to people infected with HIV.

The existence of obstacles to implementation of preventive therapy is no justification for inaction in the face of the wealth of compelling evidence supportive of preventive therapy as an essential component of disease control. Large population-based studies of preventive therapy and mathematical models both suggest that preventive treatment of tuberculosis infection, as a component of a comprehensive approach that includes active case-finding and prompt effective treatment, can produce sufficient reduction in population-level transmission and rates of active disease to interrupt the cycle of infection, illness, and death. ${ }^{20,21}$ This Series paper thus argues for an integrated approach intended to spur widespread implementation of preventive therapy in the context of a comprehensive approach to addressing the tuberculosis epidemic. We summarise the successes of preventive therapy, provide a framework for understanding new and old challenges to implementation, and lay out a pragmatic roadmap to action.

\section{Data and successes}

Isoniazid preventive therapy, which is offered in conjunction with active screening to detect and treat cases of active tuberculosis disease, has long been recognised as an effective intervention for reducing the risk of tuberculosis at both the individual and population level. From the early 1950s and during two subsequent decades, many trials assessed the efficacy of isoniazid in preventing tuberculosis in different populations and circumstances.? By 1970, overwhelming evidence from several countries had shown that isoniazid was effective for reducing the risk of tuberculosis and was safe and well tolerated in both adults and children. ${ }^{7}$ Declining incidence of tuberculosis in rural Alaska after community-wide trials of isoniazid treatment and mass screening for disease suggested a reduced force of infection attributable to mass preventive therapy during the trials. In Alaska, where tuberculosis was endemic in the 1950s, a community-wide trial of isoniazid versus placebo found a $60 \%$ decline in tuberculosis incidence for treated households that was sustained for more than two decades. ${ }^{17}$

The use of preventive therapy had a setback in the 1970s when case reports of isoniazid-associated hepatitis focused attention on the risks of preventive treatment, and a large US Public Health Service Study reported eight deaths in 13838 individuals enrolled in a trial of isoniazid, ${ }^{22}$ although subsequent analysis suggested that an unrelated epidemic of hepatitis might have contributed to the deaths recorded..$^{23}$ A series of studies using various models then argued that, for many individuals, the risks of preventive treatment outweighed the benefits. ${ }^{24}$ Of note, none of these articles considered the public health gains of preventive therapy among the benefits. 
The emergence of the worldwide HIV epidemic in the 1980 's and 1990's led to a renewed interest in prevention of tuberculosis in high-risk individuals. Several observational and randomised trials showed that using isoniazid for 6-12 months reduced the risk of tuberculosis, particularly in people with positive tuberculin skin tests. ${ }^{25}$ Guidelines developed in the USA in 2000 emphasised the importance of targeting skin testing and preventive therapy at those with highest risk of developing disease, with careful clinical monitoring to reduce toxicity. ${ }^{26}$ Yet isoniazid treatment was still littleused worldwide, including in populations with high rates of HIV infection.

The consensus on the individual-level benefit of preventive therapy is incontrovertible. Compared with untreated individuals, the risk of clinically active tuberculosis disease is reduced by $60 \%$ in immunocompetent, HIV-uninfected individuals and by $32-62 \%$ in HIV-infected adults who are treated with preventive therapy regimens of 3-12 months duration. ${ }^{25,27}$ The benefit of isoniazid preventive therapy in HIV-infected individuals is additive to the benefit of antiretroviral therapy, which itself reduces tuberculosis risk by about $60 \%$; the combination of the two can achieve substantial reductions in rates of incident tuberculosis in people with HIV infection. ${ }^{28-30}$ The risk of developing tuberculosis is reduced by about $60 \%$ in children aged 15 years or younger who receive preventive therapy. ${ }^{31}$ Long courses reduce subsequent disease in individuals at risk of exogenous re-infection. ${ }^{32}$ Finally, evidence is accumulating that preventive therapy with an appropriate drug can offer protection to individuals exposed to drugresistant tuberculosis.

At a population level, studies have built on the experience in Alaska and shown that the treatment of tuberculosis infection as part of a comprehensive tuberculosis control strategy that includes active case-finding, particularly in household contacts, can reduce tuberculosis incidence. In Rio de Janeiro, Brazil, a community-randomised trial including eight urban neighbourhoods with a baseline tuberculosis incidence of roughly 340 per 100000 people per year compared the standard procedure of informing patients with tuberculosis of the need for their household contacts to be assessed with active identification, assessment, and appropriate treatment of tuberculosis infection and tuberculosis disease in household contacts. After 5 years, tuberculosis incidence in the intervention communities was $15 \%$ lower than in the standard procedure communities. ${ }^{33}$

The cluster-randomised THRio trial in Rio de Janeiro, Brazil, involving HIV-infected people enrolled in 29 clinics, showed a benefit of isoniazid preventive treatment in a setting of moderate tuberculosis incidence, as part of an integrated care programme for HIV care. In this trial, strengthening the uptake of tuberculosis screening, tuberculin testing, and use of preventive therapy reduced the adjusted hazard of tuberculosis incidence and death in the study population by $25-30 \% .^{34}$ Long-term follow up of individuals treated with isoniazid showed that the benefit was durable for 5 years after treatment, and modelling based on the results of this intervention predicted that targeted treatment of just $20 \%$ of HIV-infected individuals with tuberculosis infection would produce a community-wide decline in HIV-related tuberculosis incidence and mortality of about $15 \% .{ }^{21,35}$

Observational evidence from other settings also supports the effectiveness of preventive therapy as a key component of achieving population-level reductions in tuberculosis incidence. To address a decade-long stagnation of tuberculosis incidence, Singapore started a new tuberculosis elimination initiative combining directly observed therapy, national surveillance, and treatment of latent infection in contacts. Tuberculosis incidence declined by $15-20 \%$ within 5 years, and treatment of latent infection was identified as a key contributor to this decline. ${ }^{36}$ In a programmatic intervention targeting two neighbourhoods with historically high tuberculosis incidence (40/100000 people per year) in Texas, USA, a door-to-door mass screening was done and isoniazid was prescribed for all infected individuals. This community-based tuberculosis screening and preventive treatment seemed to have substantially decreased subsequent tuberculosis incidence because no tuberculosis cases were detected in the two intervention communities over the next 10 years. ${ }^{37}$

\section{Challenges}

Although more than a half-century of data support the use of preventive therapy as an important component of tuberculosis control, several challenges, both new and old, should be addressed to energise widespread implementation of present guidelines and respond to perceived and actual barriers to implementation, which include clinical, technical, health systems, and policy and advocacy dimensions (table 1).

On a clinical level, practitioners encounter the difficulty of screening HIV-infected adults for tuberculosis disease before initiating preventive therapy, restricted access to tests for tuberculosis infection, the complexity of risk-factor driven algorithms to identify individuals at highest risk of disease, and the challenges of encouraging adherence to a treatment for an asymptomatic condition. These are accompanied by perceived problems such as fear of inducing drug resistance, even though evidence exists that this fear is unfounded, ${ }^{38}$ and exaggerated perceptions of the risk of severe side effects.

At the health-systems level, ambiguous or ambivalent guidelines for diagnosing and treating tuberculosis infection undermine clinician confidence. Inadequate health training and insufficient numbers of health-care workers, compounded by drug stock-outs, limit use of preventive therapy. Additionally, the absence of effective monitoring and assessment surveillance systems to oversee uptake, side effects, adherence, resistance, and 
Proposed responses

Clinical

Excluding active tuberculosis, especially in HIV-positive patients

Need for tuberculin or other testing [IGRA (interferon $\gamma$ release assay)]

Use of clinical algorithms, more use of chest $x$-rays

Develop new simple tests that are more predictive of subsequent active tuberculosis, improve worldwide production of tuberculin, treat high-risk patients without testing

Poor adherence and completion of preventive therapy Use of short-course regimens and supervision of therapy

Drug toxicity

Perceived risk of acquiring drug resistance

Encourage monthly monitoring and patient education

Health system

Available evidence suggests this is not a problem

Absence of consistent guidelines
Inadequately trained staff

Stock-outs of drugs and diagnostics (tuberculin skin test or IGRA)

Poor surveillance and reporting

Inadequate funding
Harmonised worldwide and national guidelines

Development of preventive therapy instruments

Enhanced training for doctors, nurses, and other health workers

Strengthened supply chain

Better health information systems, increased monitoring and assessment

Expansion of vertical health programmes to address tuberculosis prevention (eg, HIV prevention of mother-to-child transmission), with benchmarks for disease control; more integration of tuberculosis control into primary health care

Policy and advocacy

Absence of priority for prevention, with emphasis on proportion of active cases treated

Inadequate investment in basic, clinical and implementation research and training

Absence of advocacy and demand from groups most at risk
Realignment of tuberculosis control programmes to incorporate prevention, with performance assessment linked to incidence

Increased funding for research

Education and empowerment of at-risk group, including people with HIV and families

Table 1: Barriers to implementation of tuberculosis preventive therapy and proposed responses

programme effect make assessment of country-level efforts difficult. Further hampering use of preventive therapy is a very low level of community engagement and demand, in contrast with the demand for antiretroviral therapy and, increasingly, for treatment of hepatitis $\mathrm{C}$ virus infection. Finally, the poor prioritisation of research funding for new methods for tuberculosis control, which could include new vaccines to prevent infection and new diagnostics to predict risk of progression to disease, stifles innovation and restricts progress.

A further challenge in preventing tuberculosis is the high incidence of re-infection in HIV-infected individuals and other high-risk populations in sub-Saharan Africa, as well as other identified hotspots of uncontrolled transmission. The benefit of preventive therapy in these settings is less durable because the treatment effect seems to wane soon after discontinuation. This suggests that people treated for tuberculosis infection are either rapidly reinfected or persistent bacilli are not sterilised by isoniazid. ${ }^{32,39}$ A cluster-randomised trial done in the highburden setting of gold mines in South Africa, showed that mass screening and isoniazid preventive therapy was successful in preventing tuberculosis while individuals were receiving treatment, but had no durable effect in reducing overall tuberculosis incidence. ${ }^{40}$ Similar limitation of benefit to the time during which individuals were taking preventive therapy was noted in studies in HIV-infected individuals in Botswana and Soweto, South
Africa. ${ }^{32,41}$ Thus, although short courses of preventive therapy can provide short-term to medium-term protection even in high-burden settings, long-term protection might only be conferred where transmission is better controlled through active case-finding and treatment. Further research is needed to understand population-level effects of preventive therapy in transmission hotspots and to inform efforts to control re-infection and subsequent disease.

Solutions to most of the technical challenges mentioned previously are available and can be implemented at present. For example, if tests for infection are not available or affordable, epidemiologically targeted preventive therapy can be offered as post-exposure treatment to the highest risk individuals without evidence of active tuberculosis, such as those with HIV, child contacts of an infected person, ${ }^{42,43}$ or individuals with medical disorders that increase risk of tuberculosis. A symptom-screening algorithm with $80 \%$ sensitivity and a negative predictive value of $97 \%$ for diagnosing active tuberculosis in HIV-infected adults has been promulgated by WHO. ${ }^{44}$ In children, WHO guidance encourages simple symptom-based screening because asymptomatic children are unlikely to have active tuberculosis or to acquire drug resistance if subclinical disease is missed. ${ }^{45,46}$ The availability of cheap, rapid, and sensitive microbiological screening instruments or screening algorithms based on new tuberculosis infection diagnostics would complement efforts to scale up. 
Adherence might be improved and hepatotoxicity reduced through the use of shorter rifamycin-based preventive therapy regimens rather than the use isoniazid for long durations (ie, $6,9,12$, or 36 months). For individuals taking isoniazid, rates of drug-related liver injury can be kept low $(<1-3 \%)$ with appropriate screening and monitoring, though isoniazid-induced hepatoxicity might still result in one death for every 25000-40000 patients treated. ${ }^{40}$ In contrast, liver toxicity rates with rifamycin-based regimens are substantially lower, and adherence to these shorter course regimens is generally much better. Rifamycinbased regimen options include a weekly regimen of rifapentine plus isoniazid for 3 months, and daily regimens of isoniazid plus rifampicin or rifampicin alone for 3-4 months. ${ }^{27,41,47-49}$ These regimens are all considered options in developed countries, and are likely to have a great effect on uptake as well as the workload and health workforce needed to give and monitor preventive treatment. Further improvements in the ease of giving and taking preventive therapy are anticipated, because a new fixed-dose combination tablet of rifapentine and isoniazid is expected to be marketed later this year, and a bold 4-week regimen of daily rifapentine and isoniazid is being assessed..$^{50,51}$ Unfortunately, in resource-constrained high tuberculosis burden countries, preventive therapy with 6-36 months of isoniazid is, uninspiringly, presented as the only option. This situation is perceived as a pragmatic choice, probably because most of the historical evidence for preventive therapy is with isoniazid, which is cheap and widely available. However, the adoption of shorter, easier to complete regimens would be particularly advantageous in settings of high tuberculosis burden and limited resources because this would enable many more people to complete preventive therapy in view of the low number of health-care staff available to give treatment and monitor adverse events. Although offering real hope in addressing the adherence challenge, to be successful, these biomedical advances will have to be supported by continuous qualitative work to explore individual and community understanding of latency, its relationship to disease, and the need for treating infections with an antibiotic to prevent active disease. Understanding of how to structure tuberculosis preventive therapy programmes from the perspective of the patient is needed to design relevant interventions to promote adherence and thus a more responsive preventive therapy programme.

Further barriers to implementation affect health systems and policies. Available guidelines do not provide sufficient guidance for national preventive therapy programmes to proceed. Firstly, leadership and the responsibility for prescribing and providing preventive therapy are unclear. Ownership could rest with tuberculosis programmes, HIV programmes, primary care clinics, the private sector, or some combination of these, but a plan that outlines responsibilities and a process for coordination of efforts is necessary no matter what the arrangement. Additionally, tuberculosis programmes would need to consider further devolution of responsibilities to procure and distribute drugs for preventive therapy.

Secondly, preventive therapy has to be implemented in clinical settings with heavy workloads and programmespecific delivery targets. Although tuberculosis programmes naturally have access to high-risk groups such as household contacts, the workload of dealing with active cases often overwhelms staff, who thus deprioritise preventive therapy. Within HIV programmes, clinicians have competing priorities, such as initiating antiretroviral therapy and managing infectious and non-infectious HIVassociated comorbidities. As a result, treatment of tuberculosis infection is often de-emphasised. Training and motivating health-care workers and building systems that can undertake the additional task of providing tuberculosis preventive therapy is an important but difficult challenge. Much innovation and evidence of successful strategies is needed to address this challenge. Answers might lie in some well known but underused approaches, such as task shifting to the lay cadres of the health workforce, decentralisation of centres testing for latent tuberculosis infection, delivery of treatment and monitoring of individuals on preventive therapy, and some less explored options, such as innovative approaches for simplifying testing for latent tuberculosis infection, which could include instruments for self-testing.

Thirdly, international consensus process and outcome indicators for preventive therapy programmes are not yet available, making it difficult to assess the successes and limitations of programmes. For example, accurate country-level assessments of uptake of preventive therapy in HIV-infected people who newly presented for care have proven elusive owing to concerns with the quality of monitoring and assessment. An analysis of the uptake of preventive therapy in 150 clinical sites in South Africa suggested an increase in the absolute numbers of individuals prescribed preventive therapy (from 3309 in 2010 to 49130 in 2011) but a decrease in the proportion of patients receiving it (from 19\% in 2010 to $11 \%$ in 2011). ${ }^{52}$ Although encouraging, a reported surge in recipients is an insufficient indicator of successful implementation..$^{53}$ Indicators such as increases in coverage, completion rates, rates of adverse events, and active tuberculosis in people receiving preventive therapy are needed to show intervention fidelity. Monitoring of downward trends in the incidence of new infections and subsequent cases would provide evidence for interruption of transmission. The first Series paper ${ }^{10}$ argues for an approach in which existing data collection systems are augmented with such new information and are increasingly used to inform success of local tuberculosis control interventions such as a preventive therapy programme.

There is also a challenging shortage of advocacy and leadership to promote preventive therapy. Within Global 


\section{Reduce tuberculosis at the individual leve}

Risk-stratified preventive therapy to latently infected or exposed individuals at risk of tuberculosis, or those who might transmit future disease to susceptible people
Treat proven tuberculin-positive infection (tuberculin skin test or interferon $\gamma$ release assay positive*)
Always treat in people with HIV infection, other immune-compromised individuals, young children ( $<5$ years of age), recent skin test converters, and individuals with abnormal chest $\mathrm{x}$-rays consistent with untreated previous tuberculosis; consider treating in cigarette smokers or people with chronic lung disease, people with diabetes, malnourished individuals; recent immigrants from high-burden tuberculosis countries; health-care workers, and prisoners or residents of congregate living facilities (eg, long-term care)

Treat probable $M$ tuberculosis infection or re-infection (close contact with an infectious person)

Early diagnosis and prompt adequate treatment (also of Passive case-finding drug-resistant tuberculosis)

Active case-finding
Always treat in immune-compromised individuals (including people with HIV in a tuberculosis-endemic setting), young children ( $<5$ years of age), and household contacts with widespread exposure (all ages)

Enhanced community awareness; universal access to care; well-functioning health systems; better diagnostic instruments

Routine screening and focus on high-risk groups such as close contacts to tuberculosis patients, mine workers, prisoners, and people with HIV or diabetes

In addition to the individual approaches listed above, consider creative measures to identify transmission hot-spots and improve infection control

Ensure optimum HIV care and reduced HIV transmission, reduce cigarette smoking, indoor and outdoor air pollution, malnutrition, diabetes, alcohol, and substance misuse

*Acknowledging sensitivity and specificity limitations, not mandatory in people with HIV in a tuberculosis-endemic setting

Table 2: Treatment of $M$ tuberculosis infection as part of a comprehensive approach to improve worldwide tuberculosis control

Fund programmes and grants, tuberculosis is enormously underemphasised, which reflects countrylevel and community-level absence of advocacy and demand. In contrast, widespread availability of antiretroviral therapy has been promoted by the powerful voice of affected communities coupled with visionary leaders who devised programmes like the President's Emergency Plan for AIDS Relief (PEPFAR) and the Global Fund for AIDS, TB and Malaria. ${ }^{54}$ Building this support for tuberculosis preventive therapy will require education and engagement of both populations at risk and clinical and public health leaders. ${ }^{55}$

\section{Proposed action plan}

The abundance of existing evidence and knowledge about tuberculosis infection and preventive therapy provides a solid base for concerted worldwide action to incorporate treatment of tuberculosis infection into a comprehensive and epidemiologically sound strategy for tuberculosis elimination. Although the magnitude of the challenges implicated and the corresponding level of ambition needed are substantial, these efforts are necessary because casefinding and treatment approaches alone will not be sufficient, and both novel diagnostics to accurately identify incipient disease and effective vaccines to prevent infection or disease are distant goals. Additionally to biomedical interventions, political leadership and will are needed to modify the risk environment by addressing the social determinants of tuberculosis that perpetuate inequalities in health (see Ortblad et al, paper 4) ${ }^{56,57}$ Finally, we need a worldwide interdisciplinary approach to accelerate research that furthers our understanding of the biology of tuberculosis infection, develops novel diagnostics and drug regimens for tuberculosis infection, strengthens health systems, and enhances sustainable large scale implementation of preventive therapy programmes. In this section we provide a roadmap to address identified key implementation barriers and immediately enhance implementation.

\section{The clinical and technical approach}

Tuberculosis preventive therapy should be implemented alongside tracing of case-contacts and other high-risk individuals, targeted active case-finding, and effective treatment of active disease (see Yuen et al, paper 2 ) $^{11}$ as a routine component of tuberculosis control programmes worldwide. Commitment to preventive therapy as a core element of control is needed at the global, national, provincial, and local levels. Additionally, preventive treatment for tuberculosis should be incorporated into other health programmes that provide treatment to populations at risk, such as HIV care, substance-misuse treatment, and occupational health clinics. We propose a single-bundle strategy of routine active case finding to identify people with active disease who should be promptly initiated on effective multidrug chemotherapeutic regimens and those without disease for riskstratified treatment of $M$ tuberculosis infection (table 1, table 2). Recognising the importance of expanding preventive interventions, in 2014, WHO revised guidelines for the diagnosis and treatment of tuberculosis infection. ${ }^{55}$ The guidelines are mainly targeted at high-income or upper middle-income countries with an estimated tuberculosis incidence rate 
of less than 100 per 100000 population per year and have broadened the definitions of at-risk groups. Our proposed risk-stratified strategy aims to support these efforts and will ensure that preventive therapy is safely and efficiently provided to individuals at increased risk of disease in all settings, including high-burden countries.

In countries with uncontrolled $M$ tuberculosis transmission, re-infection might limit the long-term benefit of short courses of preventive therapy, particularly within transmission hotspots. In particularly susceptible groups, such as people with HIV, miners, prisoners in areas with high transmission rates of tuberculosis, and other groups with a high risk of developing the disease owing to occupational (eg, health-care workers) or behavioural exposures (eg, drug-users), extended or periodic schedules of preventive therapy should be implemented. $M$ tuberculosis transmission rates were astonishingly high in Alaska in the 1950s (>90\% of children were infected by the age of 15 years) when the Bethel household isoniazid study took place, and rates of infection fell precipitously as a concerted programme of case finding, treatment, and preventive therapy was implemented. ${ }^{58}$ This finding is strong evidence that preventive therapy plays an important part even in highburden areas.

\section{Health-systems, policy, and leadership}

To deliver tuberculosis preventive therapy more broadly, engagement of other health programmes that provide care to high risk populations is essential. HIV programmes can easily provide preventive therapy to HIV-infected individuals, whereas maternal and child health programmes could actively support preventive therapy provision in young and susceptible children. As the link between diabetes and tuberculosis becomes better understood, diabetes clinics and primary health programmes caring for people with diabetes could consider tuberculosis prevention, diagnosis, and treatment. Occupational health programmes are responsible for providing treatment to workers at increased risk of tuberculosis, such as miners and health-care workers, but too often they neglect prevention. In many settings, primary health centres and private practitioners can deliver preventive therapy to people who would benefit, such as contacts of individuals infected with tuberculosis disease, people with diabetes or immunosuppression, and refugees or immigrants from high-burden tuberculosis areas. To identify and standardise functional monitoring and reporting pathways is essential to support preventive therapy implementation across providers, such as latent tuberculosis registries, because this is a key driver and the only proof of actual implementation. ${ }^{59}$ In 2011 WHO launched a handbook for the programmatic management and implementation of drug-resistant tuberculosis activities and surveillance ${ }^{60} \mathrm{~A}$ similar resource is urgently needed to decode existing preventive therapy guidelines and offer practical guidance to National HIV, tuberculosis, other programme managers, monitoring and assessment coordinators, and clinicians to accelerate preventive therapy implementation in partner countries.

\section{Advocacy approach}

Widening uptake of tuberculosis preventive therapy will need leadership and evidence-based advocacy by clinicians, public health officials, and communities at risk of infection. These stakeholders should demand that preventive therapy be provided at every opportunity and contact with the health system, as it is an essential part of the tuberculosis control package. For example, for some individuals, preventive therapy will be easier to give in antiretroviral therapy clinics and antenatal programmes than through tuberculosis clinics. Additionally, the absence of understanding of tuberculosis infection and the role of preventive therapy needs to be addressed in affected individuals, their health-providers, and their communities. This education could be the key to triggering bottom-up advocacy as is seen for HIV prevention and might increase the acceptability of treatment for an asymptomatic condition.

\section{Projected population effect and cost}

Good evidence exists of the population-level effect and cost-effectiveness of preventive therapy on tuberculosis dynamics in both low-income and high-income countries. One projection for India suggests that by increasing use of preventive therapy gradually by 2050 , tuberculosis incidence could be reduced to one case per million people, and deaths could be reduced to fewer than ten cases per million people by 2035 (compared with a present estimated incidence of 1710 per million and estimated mortality of 190 per million population). ${ }^{12}$ Another projection for the Republic of Kiribati (population of roughly 100000 people, tuberculosis incidence of $487 / 100000$ people per year) suggested that a combination of active case finding and mass treatment with a full course of antituberculosis drugs given to the entire population from 2015 intermittently at 5-yearly rounds could eliminate tuberculosis from this Pacific island by $2030 .{ }^{61}$ A systematic review by Chavan and collegues provides robust evidence of the effectiveness and cost effectiveness of preventive therapy in high-income countries. Remarkably, the analysis concluded that tuberculosis preventive therapy would be effective and cost effective even for adults up to aged 80 years old. ${ }^{62}$

\section{Conclusions}

After more than three decades of policy that focused only on the detection and treatment of active cases of tuberculosis, a better understanding of the epidemiology and population dynamics of the disease has emerged, and the essential role of controlling the seedbeds of disease (asymptomatic tuberculosis infections) is now understood. Evidence of the effectiveness of preventive therapy in high-risk individuals is abundant, and proof of 
the population-level effect of preventive therapy exists in many settings across the globe. Implementation of tuberculosis preventive therapy will need addressing of clinical, administrative, structural, and economic barriers, and the engagement of several sectors, not just national tuberculosis programmes. With the advent of new treatments that shorten and simplify preventive therapy, the ability of health systems to reach and treat more highrisk individuals will be enhanced. As with malaria, HIV and other infectious diseases of public health consequence, the key role of preventive therapy as part of a comprehensive control strategy for tuberculosis must be recognised and executed.

\section{Contributors}

REC and MXR conceived the idea for this Series paper. REC and MXR wrote the first draft of the paper, and all authors revised it for important intellectual content. All authors approved the final version submitted for publication.

\section{Declaration of interests}

We declare no competing interests. The content is solely the responsibility of the authors and does not necessarily represent the views of the National Institutes of Health.

\section{Acknowledgments}

We thank Ed Nardell for his review and the important comments he contributed during drafting, and Carly Rodriguez for coordination and research assistance in the preparation of this manuscript. MXR was supported by a University College London Excellence Fellowship and reports grants from the National Institute for Health Research University College London Hospitals Biomedical Research Centre. NAM reports grants from the US National Institutes of Health (R01 HD064354-03 and R01 DA030276-01A1) and the Medical Research Council Soweto Matlosana Center for HIV/AIDS and TB Research, and NAM and REC report grants from the US Centers for Disease Control and Prevention (2000-2009-32589). REC reports grants from the US National Institutes of Health (P30-AI-094189).

\section{References}

1 World Health Organization. Global strategy and targets for tuberculosis prevention, care and control after 2015. http://apps. who.int/gb/ebwha/pdf_files/EB134/B134_12-en.pdf?ua=1 (accessed July 25, 2015).

2 Barry CE 3rd, Boshoff HI, Dartois V, et al. The spectrum of latent tuberculosis: rethinking the biology and intervention strategies. Nat Rev Microbiol 2009; 7: 845-55.

3 Ernst JD. The immunological life cycle of tuberculosis. Nat Rev Immunol 2012; 12: 581-91.

4 Getahun H, Matteelli A, Chaisson RE, Raviglione M. Latent Mycobacterium tuberculosis infection. N Engl J Med 2015; 372: 2127-35.

5 Ewer K, Millington KA, Deeks JJ, Alvarez L, Bryant G, Lalvani A. Dynamic antigen-specific T-cell responses after point-source exposure to Mycobacterium tuberculosis. Am J Respir Crit Care Med 2006; 174: 831-39.

6 Dowdy DW, Chaisson RE. The persistence of tuberculosis in the age of DOTS: reassessing the effect of case detection. Bull World Health Organ 2009; 87: 296-304.

7 Ferebee SH. Controlled chemoprophylaxis trials in tuberculosis. A general review. Bibl Tuberc 1970; 26: 28-106.

8 Dye C, Scheele S, Dolin P, Pathania V, Raviglione MC. Consensus statement. Global burden of tuberculosis: estimated incidence, prevalence, and mortality by country. WHO Global Surveillance and Monitoring Project. JAMA 1999; 282: 677-86.

9 Gao L, Lu W, Bai L, et al. Latent tuberculosis infection in rural China: baseline results of a population-based, multicentre, prospective cohort study. Lancet Infect Dis 2015; 15: 310-19.

10 Theron G, Jenkins HE, Cobelens F, et al. Data for action: collection and use of local data to end tuberculosis. Lancet 2015; published online Oct 26. http://dx.doi.org/10.1016/S0140-6736(15)00321-9.
11 Yuen CM, Amanullah F, Dharmadhikari A, et al. Turning off the tap: stopping tuberculosis transmission through active case-finding and prompt effective treatment. Lancet 2015; published online Oct 26. http://dx.doi.org/10.1016/S0140-6736(15)00322-0.

12 Dye C, Glaziou P, Floyd K, Raviglione M. Prospects for tuberculosis elimination. Annu Rev Public Health 2013; 34: 271-86.

13 Henderson DA. Principles and lessons from the smallpox eradication programme. Bull World Health Org 1987; 65: 535-46.

14 Mackenzie CD, Homeida MM, Hopkins AD, Lawrence JC. Elimination of onchocerciasis from Africa: possible? Trends Parasitol 2012; 28: 16-22.

15 Rangaka MX, Wilkinson KA, Glynn JR, et al. Predictive value of interferon-gamma release assays for incident active tuberculosis: a systematic review and meta-analysis. Lancet Infect Dis 2012; 12: $45-55$.

16 Lincoln EM. The effect of antimicrobial therapy on the prognosis of primary tuberculosis in children. Am Rev Tuberc 1954; 69: 682-89.

17 Comstock GW, Baum C, Snider DE Jr. Isoniazid prophylaxis among Alaskan Eskimos: a final report of the bethel isoniazid studies. Am Rev Respir Dis 1979; 119: 827-30.

18 Egmose T, Ang'awa JOW, Poti SJ. The use of isoniazid among household contacts of open cases of pulmonary tuberculosis. Bull World Health Org 1965; 33: 419-33.

19 Stop TB. Guidelines for intensified tuberculosis case-finding and isoniazid preventive therapy for people living with HIV in resource-constrained settings. Geneva, Switzerland: World Health Organization, 2011.

20 Mills HL, Cohen T, Colijn C. Modelling the performance of isoniazid preventive therapy for reducing tuberculosis in HIV endemic settings: the effects of network structure. J R Soc Interface 2011; 8: 1510-20.

21 Dowdy DW, Golub JE, Saraceni V, Moulton LH, Cavalcante SC, Cohn S, et al. Impact of isoniazid preventive therapy for HIV-infected adults in Rio de Janeiro, Brazil: an epidemiological model. J Acquir Immune Defic Syndr 2014; 66: 552-58.

22 Kopanoff DE, Snider DE Jr, Caras GJ. Isoniazid-related hepatitis: a US Public Health Service cooperative surveillance study. Am Rev Respir Dis 1978; 117: 991-1001.

23 Comstock GW. Prevention of tuberculosis among tuberculin reactors: maximizing benefits, minimizing risks. JAMA 1986; 256: $2729-30$.

24 Tsevat J, Taylor WC, Wong JB, Pauker SG. Isoniazid for the tuberculin reactor: take it or leave it. Am Rev Respir Dis 1988; 137: 215-20.

25 Akolo C, Adetifa I, Shepperd S, Volmink J. Treatment of latent tuberculosis infection in HIV infected persons. Cochrane Database Syst Rev 2010; 20: CD000171.

26 Targeted tuberculin testing and treatment of latent tuberculosis infection. This official statement of the American Thoracic Society was adopted by the ATS Board of Directors, July 1999. This is a Joint Statement of the American Thoracic Society (ATS) and the Centers for Disease Control and Prevention (CDC). This statement was endorsed by the Council of the Infectious Diseases Society of America. (IDSA), September 1999, and the sections of this statement. Am J Respir Crit Care Med 2000; 161: S221-47.

27 Smieja MJ, Marchetti CA, Cook DJ, Smaill FM. Isoniazid for preventing tuberculosis in non-HIV infected persons. Cochrane Database Syst Rev 2000; 2: CD001363.

28 Golub JE, Saraceni V, Cavalcante SC, et al. The impact of antiretroviral therapy and isoniazid preventive therapy on tuberculosis incidence in HIV-infected patients in Rio de Janeiro, Brazil. AIDS 2007; 21: 1441-8.

29 Rangaka MX, Wilkinson RJ, Boulle A, et al. Isoniazid plus antiretroviral therapy to prevent tuberculosis: a randomised double-blind, placebo-controlled trial. Lancet 2014; 384: 682-90.

30 TEMPRANO ANRS 12136 Study Group. A trial of early antiretrovirals and isoniazid preventive therapy in Africa. N Engl J Med 2015; 373: 808-22.

31 Ayieko J, Abuogi L, Simchowitz B, Bukusi EA, Smith AH, Reingold A. Efficacy of isoniazid prophylactic therapy in prevention of tuberculosis in children: a meta-analysis. BMC Infect Dis 2014; 14: 91

32 Samandari T, Agizew TB, Nyirenda S, et al. 6-month versus 36-month isoniazid preventive treatment for tuberculosis in adults with HIV infection in Botswana: a randomised, double-blind, placebocontrolled trial. Lancet 2011; 377: 1588-98. 
33 Cavalcante SC, Durovni B, Barnes GL, et al. Community-randomized trial of enhanced DOTS for tuberculosis control in Rio de Janeiro, Brazil. Int J Tuberc Lung Dis 2010; 14: 203-09.

34 Durovni B, Saraceni V, Moulton LH, et al. Effect of improved tuberculosis screening and isoniazid preventive therapy on incidence of tuberculosis and death in patients with HIV in clinics in Rio de Janeiro, Brazil: a stepped wedge, cluster-randomised trial. Lancet Infect Dis 2013; 13: 852-58.

35 Golub JE, Cohn S, Saraceni V, et al. Long-term protection from isoniazid preventive therapy for tuberculosis in HIV-infected patients in a medium-burden tuberculosis setting: the TB/HIV in Rio (THRio) study. Clin Infect Dis 2015; 60: 639-45.

36 Chee CB, Teleman MD, Boudville IC, Do SE, Wang YT. Treatment of latent TB infection for close contacts as a complementary TB control strategy in Singapore. Int J Tuberc Lung Dis 2004; 8: 226-31.

37 Cegielski JP, Griffith DE, McGaha PK, et al. Eliminating tuberculosis one neighborhood at a time. Am J Public Health 2013; 103: $1292-300$

38 Balcells ME, Thomas SL, Godfrey-Faussett P, Grant AD. Isoniazid preventive therapy and risk for resistant tuberculosis. Emerg Infect Dis 2006; 12: 744-51.

39 Dickinson JM, Mitchison DA. Experimental models to explain the high sterilizing activity of rifampin in the chemotherapy of tuberculosis. Am Rev Respir Dis 1981; 123: 367-71.

40 Churchyard GJ, Fielding KL, Lewis JJ, et al. A trial of mass isoniazid preventive therapy for tuberculosis control. N Engl J Med 2014; 370: 301-10.

41 Martinson NA, Barnes GL, Moulton LH, et al. New regimens to prevent tuberculosis in adults with HIV infection. $N$ Engl J Med 2011; 365: 11-20.

42 Triasih R, Robertson CF, Duke T, Graham SM. A prospective evaluation of the symptom-based screening approach to the management of children who are contacts of tuberculosis cases. Clin Infect Dis 2015; 60: 12-18.

43 Mandalakas AM, Kirchner HL, Lombard C, et al. Well-quantified tuberculosis exposure is a reliable surrogate measure of tuberculosis infection. Int J Tuberc Lung Dis 2012 Aug; 16: 1033-9

44 Getahun H KW, Heilig CM, Corbett EL, et al. Development of a standardized screening rule of tuberculosis in people living with HIV in resource-constrained settings: Individual participant data meta-analysis of observational studies. PLoS Med 2011; 8: 1-13.

45 WHO. Guidance for National Tuberculosis Programmes on the management of tuberculosis in children - second edition. Geneva Switzerland: Stop TB Partnership Childhood TB Subgroup, World Health Organization, 2014.

46 Kruk A, Gie RP, Schaaf HS, Marais BJ. Symptom-based screening of child tuberculosis contacts: improved feasibility in resource-limited settings. Pediatrics 2008; 121: e1646-52

47 Sterling TR, Villarino ME, Borisov AS, et al. Three months of rifapentine and isoniazid for latent tuberculosis infection. N Engl J Med 2011; 365: 2155-66.
48 Villarino ME, Scott NA, Weis SE, et al. Treatment for preventing tuberculosis in children and adolescents: a randomized clinical trial of a 3-month, 12-dose regimen of a combination of rifapentine and isoniazid. JAMA Pediatr 2015; 169: 247-55.

49 Centers for Disease C, Prevention. Recommendations for use of an isoniazid-rifapentine regimen with direct observation to treat latent Mycobacterium tuberculosis infection. MMWR Morb Mortal Wkly Rep 2011; 60: 1650-53.

50 Zhang T, Zhang M, Rosenthal IM, Grosset JH, Nuermberger EL. Short-course therapy with daily rifapentine in a murine model of latent tuberculosis infection. Am J Respir Crit Care Med 2009; 180: 1151-57.

51 Podany AT, Bao Y, Chaisson RE, et al. Efavirenz pharmacokinetics in HIV-infected persons receiving rifapentine and isoniazid for tuberculosis prevention. Clin Infect Dis 2015; published online June 16. DOI:10.1093/cid/civ464.

52 Bristow CC, Larson E, Vilakazi-Nhlapo AK, Wilson M, Klausner JD. Scale-up of isoniazid preventive therapy in PEPFAR-assisted clinical sites in South Africa. Int J Tuberc Lung Dis 2012; 16: 1020-22.

53 Martinson NA, McLeod KE, Milovanovic M, Msandiwa R, Lebina L. Implementation of isoniazid preventive therapy for HIV-infected adults: overstatement of district reports. Int J Tuberc Lung Dis 2014; 18: 1005 .

54 Keshavjee S, Beauvais SG. Let's learn from HIV activists how to achieve zero tuberculosis deaths. Huffington Post. http://www. huffingtonpost.com/salmaan-keshavjee/tb-hiv-patients-awareness_ b_1700450.html (accessed Sept 19, 2015).

55 WHO. Guidelines on the management of latent tuberculosis infection. Geneva, Switzerland: World Health Organization, 2015.

56 Ortblad KF, Salomon JA, Bärnighausen T, Atun R. Stopping tuberculosis: a biosocial model for sustainable development. Lancet 2015; published online Oct 26. http://dx.doi.org/10.1016/S01406736(15)00324-4.

57 Raviglione M, Krech R. Tuberculosis: still a social disease. Int J Tuberc Lung Dis 2011; 15 (suppl 2): S6-8.

58 Kaplan GJ, Fraser RI, Comstock GW. Tuberculosis in Alaska, 1970 the continued decline of the tuberculosis epidemic. Am Rev Respir Dis 1972; 105: 920-26.

59 van Soelen N, du Preez K, van Wyk SS,et al. Does an isoniazid prophylaxis register improve tuberculosis contact management in South African children? PLoS One 2013; 8: e80803.

60 WHO. Guidelines for the programmatic management of drugresistant tuberculosis 2011 update. Geneva, Switzerland: World Health Organization, 2011.

61 Hill PC, Dye C, Viney K, et al. Mass treatment to eliminate tuberculosis from an island population. Int J Tuberc Lung Dis 2014; 18: 899-904.

62 Chavan S, Newlands D, Smith C. A systematic review of economic evaluations of chemoprophylaxis for tuberculosis. J Trop Med 2011; 2011: 130976 